\title{
ON NONLINEAR OVERSTABLE CONVECTION ROLLS IN A ROTATING SYSTEM
}

\author{
N. RIAHI' \\ (Received 16 December 1980; revised 30 July 1982)
}

\begin{abstract}
Finite amplitude oscillatory convection rolls in the form of travelling waves are studied for a horizontal layer of a low Prandtl number fluid heated from below and rotating rapidly about a vertical axis. The results of the stability and nonlinear analyses indicate that there is no subcritical instability and that the oscillatory rolls are unstable for the ranges of the Prandtl number and the rotation rate considered in this paper.
\end{abstract}

\section{Introduction}

This paper studies finite amplitude oscillatory convection rolls in the form of travelling waves and the subsequent stability analysis in a horizontally infinite layer of fluid with a low Prandtl number $P$ rotating rapidly about a vertical axis. Since $P$ is small and the rotation parameter $\tau$ is large, the onset of convection occurs in the form of an oscillatory mode (Chandrasekhar [2]). The subsequent nonlinear time dependent and stability problem of the present investigation is solved using a perturbation method similar to that initiated by Schluter et al. [8]. Using the method of Schluter et al., Kuppers and Lortz [5] and Kuppers [4] solved the finite amplitude steady problem in the rotating case and found that all the steady solutions are unstable for $\tau$ beyond a critical value, $\tau_{c}$. Hence, if for $\tau>\tau_{c}$ the Rayleigh number $R$ is increased from a subcritical value, then there is a transition from pure conduction to a time dependent convective flow. The problem of the physically realized solutions for $P>1$ (where oscillatory motion is not possible at the onset of convection [2]) was attacked by Busse and Clever [1].

\footnotetext{
'Department of Theoretical and Applied Mechanics, University of Illinois at Urbana-Champaign, Urbana, Illinois 61801, U.S.A.

(c) Copyright Australian Mathematical Society 1984, Serial-fee code 0334-2700/84
} 
They demonstrated that time dependent solutions in the form of periodic nonlinear oscillations do indeed exist which depend on the initial conditions as well as on the spectrum of small disturbances. They also pointed out that their theoretical model represents a manifold of solutions which are all unstable and replace each other continuously. The problem of convection in a rotating fluid that offers a simple model exhibiting such a basic property of turbulence is, therefore, of theoretical interest for further understanding the features of turbulent fluid systems.

The present study is concerned with another kind of time dependent flow: the finite amplitude development of the linear effect of the so-called overstability. For the case of the time dependent convection rolls in the form of travelling waves, the nonlinear properties of the motion for the Rayleigh number $R$ close to its critical value $R_{0 c}$ (based on the linear theory) is studied and the stability of the oscillatory rolls with respect to all the disturbances in the form of rolls which are inclined at arbitrary angles to the original oscillatory rolls are investigated. All such disturbances are found to lead to instability, and the disturbances which are along the original rolls are shown to have the largest growth rate. Such disturbances are expected to be predominant in the subsequent nonlinear development of the convective flow. Another important result of the present study is that no subcritical instability is found for the ranges of $P$ and $\tau$ considered in this paper. Hence, the finite amplitude oscillatory convection rolls exist only for $R>R_{\text {oc }}$.

\section{Oscillatory convective motion}

We consider an infinite horizontal layer of a low Prandtl number fluid (such as mercury) of depth $d$ bounded above and below by two stress-free perfectly conducting planes maintained at temperatures $T_{0}$ and $T_{0}+\Delta T$ (with $\Delta T>0$ ). The fluid is rotating about the vertical axis with a constant angular velocity $\Omega$. It is known, [3], that, under the usual Boussinesq approximation, the non-dimensional forms of the equations for momentum, heat and conservation of mass can be simplified by using the general representation

$$
\begin{gathered}
\mathbf{u}=\delta v+\epsilon \psi, \\
\delta=\nabla \times \nabla \times \lambda, \quad \epsilon=\nabla \times \lambda,
\end{gathered}
$$

for the velocity field $u$. Here $\lambda$ represents a unit vector in the vertical direction, and $v$ and $\psi$ are two scalar functions. The components $\delta v$ and $\epsilon \psi$ for $u$ in (la) are called the poloidal and toroidal components of the velocity vector, respectively. Taking the vertical components of the curl and curl of the curl of the momentum 
equation and using (1) in the heat equation yields the following governing equations:

$$
\begin{gathered}
\Delta_{2}\left[\nabla^{2}\left(\nabla^{2}-\frac{\partial}{\partial t}\right) v-\tau \frac{\partial \psi}{\partial z}-\theta\right]=\delta \cdot[(\delta v+\epsilon \psi) \cdot \nabla(\delta v+\epsilon \psi)], \\
\Delta_{2}\left[\left(\nabla^{2}-\frac{\partial}{\partial t}\right) \psi+\tau \frac{\partial v}{\partial z}\right]=\epsilon \cdot[(\delta v+\epsilon \psi) \cdot \nabla(\delta v+\epsilon \psi)], \\
\left(\nabla^{2}-P \frac{\partial}{\partial t}\right) \theta-R \Delta_{2} v=P(\delta v+\epsilon \psi) \cdot \nabla \theta
\end{gathered}
$$

Here $\theta$ is the derivation of temperature from its static value, $P=\nu / D$ is the Prandtl number, $\nu$ is the kinematic viscosity, $D$ is the thermal diffusivity, $R=\alpha g \Delta T d^{3} /(D \nu)$ is the Rayleigh number, $\alpha$ is the coefficient of thermal expansion, $g$ is the acceleration due to gravity, $\tau=2 \Omega d^{2} / \nu$ is the rotation parameter (square root of the Taylor number), $t$ is the time variable, and $\Delta_{2}$ is the horizontal Laplacian. The notations and equations introduced above are now standard in thermal convection theory, and the reader is referred to [3] for some details regarding these matters. The boundary conditions appropriate to stress-free conducting surfaces at $z= \pm 1 / 2$ are known, [3], to be

$$
v=\partial^{2} v / \partial z^{2}=\partial \psi / \partial z=\theta=0 \text {. }
$$

The stability of the solutions $v, \psi, \theta$ of (2)-(3) is investigated by superposing on the solutions perturbations $\tilde{v}, \tilde{\psi}, \tilde{\theta}$ of infinitesimal amplitude and with addition of a time dependence of the form $\exp (\sigma t)$, where $\sigma$ is the growth rate. When the governing equations and boundary conditions (2)-(3) are subtracted from the corresponding equations and boundary conditions for the total dependent variables plus perturbation quantities and the resulting system is linearized with respect to the perturbation quantities, we obtain the following linear stability equations

$$
\begin{aligned}
\Delta_{2}\left[\nabla ^ { 2 } \left(\nabla^{2}\right.\right. & \left.\left.-\sigma \frac{\partial}{\partial t}\right) \tilde{v}-\tau \frac{\partial \tilde{\psi}}{\partial z}-\tilde{\theta}\right] \\
& =\delta \cdot[(\delta \tilde{v}+\epsilon \tilde{\psi}) \cdot \nabla(\delta v+\epsilon \psi)+(\delta v+\epsilon \psi) \cdot \nabla(\delta \tilde{v}+\epsilon \tilde{\psi})]
\end{aligned}
$$

$$
\begin{aligned}
\Delta_{2}\left[\left(\nabla^{2}-\sigma\right.\right. & \left.\left.-\frac{\partial}{\partial t}\right)+\tau \frac{\partial \tilde{v}}{\partial z}\right] \\
& =\epsilon \cdot[(\delta \tilde{v}+\epsilon \tilde{\psi}) \cdot \nabla(\delta v+\epsilon \psi)+(\delta v+\epsilon \psi) \cdot \nabla(\delta \tilde{v}+\epsilon \tilde{\psi})],
\end{aligned}
$$

$$
\left(\nabla^{2}-P \sigma-P \frac{\partial}{\partial t}\right) \tilde{\theta}-R \Delta_{2} \tilde{v}=P[(\delta \tilde{v}+\epsilon \tilde{\psi}) \cdot \nabla \theta+(\delta v+\epsilon \psi) \cdot \nabla \tilde{\theta}]
$$


and the appropriate boundary conditions at $z= \pm 1 / 2$ are then clearly

$$
\tilde{v}=\partial^{2} \tilde{v} / \partial z^{2}=\partial \tilde{\psi} / \partial z=\tilde{\theta}=0 .
$$

The systems (2)-(3) and (4)-(5) are analyzed by a method of small amplitude perturbation similar to that initiated by Schluter et al. [8]. The dependent variables, $R$ and $\omega$ (frequency of the overstable motion) are expanded with respect to an amplitude parameter $\mu$,

$$
(v, \psi, \theta)=\sum_{m=1}^{\infty} \mu^{m}\left(v_{m}, \psi_{m}, \theta_{m}\right), \quad(R, \omega)=\sum_{m=0}^{\infty} \mu^{m}\left(R_{m}, \omega_{m}\right) .
$$

Using (6) in (2)-(3) yields a set of inhomogeneous systems the solvability conditions of which determine $R_{m}$ and $\omega_{m}$. As usual, these conditions mean that the solutions of the inhomogeneous equations should be orthogonal to the solutions of the adjoint homogeneous equations. Similarly, the stability equations are solved by expanding $\tilde{v}, \tilde{\psi}, \tilde{\theta}$ and $\sigma$ with respect to $\mu$ as

$$
(\tilde{v}, \tilde{\psi}, \tilde{\theta})=\sum_{m=1}^{\infty} \mu^{m-1}\left(\tilde{v}_{m}, \tilde{\psi}_{m}, \tilde{\theta}_{m}\right), \quad \sigma=\sum_{m=0}^{\infty} \mu^{m} \sigma_{m}
$$

The method of finite amplitude and stability analysis carried out in the present study is of fairly standard nature ([8], [5]). Furthermore, the algebra involved in the solutions of (2)-(3) and (4)-(5) is so extremely tedious and lengthy that only the solutions to the lowest order in $\mu$ and the method of procedure will be outlined here. The solutions $v_{m}, \psi_{m}, \theta_{m}(m>1)$ and $\tilde{v}_{m}, \tilde{\psi}_{m}, \tilde{\theta}_{m}$ will not be written explicitly.

The basic model treated in this paper is for a rapidly rotating system such that $P$ lies in the range

$$
\tau^{-1} \ll P \ll \tau^{-2 / 5} .
$$

The assumption (8) simplifies considerably the lengthy algebra involved in the solutions of the nonlinear problem and is also physically realistic. It involves, as it will be discussed in the next section, a rapidly rotating layer of a fluid such as mercury.

By inserting (6) into (2)-(3) and disregarding the quadratic terms, we find that the solutions of the linear system for the two-dimensional rolls, to the leading order terms, can be written as

$$
\left(v_{1}, \psi_{1}, \theta_{1}\right)=\left[f_{s}(z), g_{s}(z), h_{s}(z)\right] \sum_{m=-1}^{1} c_{m}\left[f_{t}\left(\gamma_{m}^{+}\right), g_{t}\left(\gamma_{m}^{+}\right), h_{t}\left(\gamma_{m}^{+}\right)\right]
$$

where

$$
\gamma_{m}^{+}=\mathbf{k}_{m} \cdot \mathbf{r}+\omega_{0} t
$$




$$
\begin{gathered}
f_{s}(z)=\sqrt{2} \cos \pi z, \quad g_{s}(z)=\omega_{0}^{-1} \tau \frac{d f_{s}}{d z}, \\
h_{s}(z)=2 a^{2} f_{s}, \\
f_{t}\left(t^{*}\right)=\cos t^{*}, \quad g_{t}\left(t^{*}\right)=\sin t^{*}+\omega_{0}^{-1} a^{2} \cos t^{*}, \\
h_{t}\left(t^{*}\right)=\cos t^{*}+a^{-1} P \omega_{0} \sin t^{*},
\end{gathered}
$$

$\mathbf{r}$ is the horizontal position vector, $\mathbf{k}_{m}$ are horizontal wave number vectors satisfying $\left|\mathbf{k}_{m}\right|=a$, and $c_{m}$ are constants. The quantities $R_{0}$ and $\omega_{0}^{2}$ which are functions of $P, \tau$ and the wave number $a$, are found from a characteristic equation derived from ( 9 ) and the linear system of equations, and they can be written in the following forms:

$$
\begin{gathered}
R_{0}=2 a^{4}+2(P \tau \pi)^{2} a^{-2}, \\
\omega_{0}^{2}=\pi^{2} \tau^{2} a^{-2}-a^{4} .
\end{gathered}
$$

These results are well known ([2]). The critical values of $R_{0}, \omega_{0}^{2}$ and $a$ for which $R_{0}$ is minimized with respect to $a$ are

$$
R_{0 c}=3.2^{1 / 3}(P \tau \pi)^{4 / 3}, \quad \omega_{0 c}^{2}=a_{c}^{-2} \pi^{2} \tau^{2}, \quad a_{c}=(P \tau \pi / 2)^{1 / 3} .
$$

In the following, we assume $a=a_{c}$ because we are mainly concerned in the present study with the weakly nonlinear oscillatory motion of convection rolls rather than the range of the wave numbers of the realized convection flows. We also restrict ourselves to those disturbances with the wave numbers $\tilde{a}=a_{c}$ and frequencies $\tilde{\omega}=\omega_{c}$. Then the most critical disturbance which has the maximum growth rate is found to be characterized by $\sigma_{0}=0$. By inserting (6)-(7) into (4)-(5) and disregarding the quadratic terms, we find that the solutions of the linear system for the disturbances, to the leading order terms, can be written as

$$
\begin{aligned}
& \left(\tilde{v}_{1}, \tilde{\psi}_{1}, \tilde{\theta}_{1}\right)=\left[f_{s}(z), g_{s}(z), h_{s}(z)\right] \\
& \quad \times \sum_{m=1}^{1}\left\{\tilde{c}_{m}\left[f_{t}\left(\gamma_{m}^{+}\right), g_{t}\left(\gamma_{m}^{+}\right), h_{t}\left(\gamma_{m}^{+}\right)\right]+\tilde{E}_{m}\left[\hat{f}_{t}\left(\gamma_{m}^{+}\right), \hat{g}_{t}\left(\gamma_{m}^{+}\right), \hat{h}_{t}\left(\gamma_{m}^{+}\right)\right]\right\},
\end{aligned}
$$

where

$$
\begin{aligned}
& \hat{f}_{t}\left(\gamma_{m}^{+}\right)=\sin \left(\gamma_{m}^{+}\right), \quad \hat{g}_{t}\left(\gamma_{m}^{+}\right)=-\cos \left(\gamma_{m}^{+}\right)+\omega_{0}^{-1} a^{2} \sin \left(\gamma_{m}^{+}\right) \\
& \hat{h}_{t}\left(\gamma_{m}^{+}\right)=\sin \left(\gamma_{m}^{+}\right)-a^{2} p \omega_{0} \cos \left(\gamma_{m}^{+}\right)
\end{aligned}
$$

and $\tilde{c}_{m}$ and $\tilde{E}_{m}$ are arbitrary constants.

In contrast to the steady problem treated by Kuppers and Lortz [5], the linear system in the present time dependent problem is not self adjoint, and the 
solvability conditions to be formed next require us to know two independent particular solutions of the adjoint linear system

$$
\begin{gathered}
\Delta_{2}\left[\nabla^{2}\left(\nabla^{2}+\frac{\partial}{\partial t}\right) v-\tau \frac{\partial \psi}{\partial z}-\theta\right]=0, \\
\Delta_{2}\left[\left(\nabla^{2}+\frac{\partial}{\partial t}\right) \psi+\tau \frac{\partial v}{\partial z}\right]=0, \\
\left(\nabla^{2}+P \frac{\partial}{\partial t}\right) \theta-R \Delta_{2} v=0,
\end{gathered}
$$

which satisfy the boundary conditions (3). These are (labelled by the subscripts 11 and 12):

$$
\begin{aligned}
& \left(v_{11}, \psi_{11}, \theta_{11}\right)=\left[f_{s}(z) f_{t}\left(\gamma_{m}^{-}\right), g_{s}(z) g_{t}\left(\gamma_{m}^{-}\right), h_{s}(z) h_{t}\left(\gamma_{m}^{-}\right)\right], \\
& \left(v_{11}, \psi_{12}, \theta_{12}\right)=\left[f_{s}(z) \hat{f}_{t}\left(\gamma_{m}^{-}\right), g_{s}(z) \hat{g}_{t}\left(\gamma_{m}^{-}\right), h_{s}(z) \hat{h}_{t}\left(\gamma_{m}^{-}\right)\right],
\end{aligned}
$$

where

$$
\gamma_{m}^{-}=\mathbf{k}_{m} \cdot \mathbf{r}-\omega_{0} t .
$$

The solvability conditions for the second order system of equations in $\mu$ for the oscillatory rolls which are given below are derived after multiplying the momentum equations for the poloidal and toroidal components of velocity by $v_{1 /}$ and $\psi_{1 I}$ $(I=1,2)$, the heat equation by $R_{0}^{-1} \theta_{1 I}$, adding and integrating over the whole volume of fluid, averaging in time over a period of $2 \pi / \omega_{0}$, and using the appropriate boundary conditions. They are

$$
\begin{aligned}
R_{0}^{-1} R_{1}\left\langle\theta_{1 I} \Delta_{2} v_{1}\right\rangle+\omega_{0}^{-1} \omega_{1}\left\langle v_{1 I} \nabla^{2} \Delta_{2} \frac{\partial v_{1}}{\partial t}+\psi_{1 I} \Delta_{2} \frac{\partial \psi_{1}}{\partial t}+R_{0}^{-1} P \theta_{1 I} \frac{\partial \theta_{1}}{\partial t}\right\rangle & \\
+\left\langle v_{1 I} \boldsymbol{\delta} \cdot\left(\mathbf{u}_{1} \cdot \nabla \mathbf{u}_{1}\right)+\psi_{1 I} \epsilon \cdot\left(\mathbf{u}_{1} \cdot \nabla \mathbf{u}_{1}\right)+R_{0}^{-1} P \theta_{1 I}\left(\mathbf{u}_{1} \cdot \nabla \theta_{1}\right)\right\rangle & =0, \\
I & =1,2 .
\end{aligned}
$$

In a similar manner, the solvability conditions for the second order system of equations in $\mu$ for the disturbances are derived and are given below.

$$
\begin{aligned}
& 0=\left\langle v_{1 I} \nabla^{2} \Delta_{2}\left(\sigma_{1}+\omega_{0}^{-1} \omega_{1} \frac{\partial}{\partial t}\right) \tilde{v}_{1}+\psi_{1 I} \Delta_{2}\left(\sigma_{1}+\omega_{0}^{-1} \omega_{1} \frac{\partial}{\partial t}\right) \tilde{\psi}_{1}\right. \\
& \left.+R_{0}^{-1} P \theta_{1 I}\left(\sigma_{1}+\omega_{0}^{-1} \omega_{1} \frac{\partial}{\partial t}\right) \tilde{\theta}_{1}\right\rangle+R_{0}^{-1} R_{1}\left\langle\theta_{1 /} \Delta_{2} \tilde{v}_{1}\right\rangle \\
& +\left\langle v_{1 /} \delta \cdot\left(\mathbf{u}_{1} \cdot \nabla \tilde{\mathbf{u}}_{1}+\tilde{\mathbf{u}}_{1} \cdot \nabla \mathbf{u}_{1}\right)\right. \\
& \left.+\psi_{1}, \epsilon \cdot\left(\mathbf{u}_{1} \cdot \nabla \tilde{\mathbf{u}}_{1}+\tilde{\mathbf{u}}_{1} \cdot \nabla \mathbf{u}_{1}\right)+R_{0}^{-1} P \theta_{1 /}\left(\mathbf{u}_{1} \cdot \nabla \tilde{\theta}_{1}+\tilde{\mathbf{u}}_{1} \cdot \nabla \theta_{1}\right)\right\rangle, \quad I=1,2 .
\end{aligned}
$$


The triple products in (15) are zero due to the symmetry of the function $f_{s}(z)$. The equations (15) then yield

$$
R_{1}=\omega_{1}=\sigma_{1}=0 .
$$

The solvability conditions for the third order systems of equations in $\mu$ for the oscillatory rolls and the disturbances which are given below are derived by direct analogy to that described above for the second order systems of equations in $\mu$.

$$
\begin{gathered}
R_{0}^{-1} R_{2}\left\langle\theta_{1 I} \Delta_{2} v_{1}\right\rangle+\omega_{0}^{-1} \omega_{2}\left\langle v_{1 I} \nabla^{2} \Delta_{2} \frac{\partial v_{1}}{\partial t}+\psi_{1 I} \Delta_{2} \frac{\partial \psi_{1}}{\partial t}+R_{0}^{-1} P \theta_{1 I} \frac{\partial \theta_{1}}{\partial t}\right\rangle \\
+\left\langle\left(v_{1 I} \boldsymbol{\delta}+\psi_{1 I} \epsilon\right) \cdot\left(\mathbf{u}_{1} \cdot \nabla \mathbf{u}_{2}+\mathbf{u}_{2} \cdot \nabla \mathbf{u}_{1}\right)\right. \\
\left.+R_{0}^{-1} P \theta_{1 I}\left(\mathbf{u}_{1} \cdot \nabla \theta_{2}+\mathbf{u}_{2} \cdot \nabla \theta_{1}\right)\right\rangle=0, \quad I=1,2, \\
\left\langle v_{1 I} \nabla^{2} \Delta_{2}\left(\sigma_{2}+\omega_{0}^{-1} \omega_{2} \frac{\partial}{\partial t}\right) \tilde{v}_{1}+\psi_{1 I} \Delta_{2}\left(\sigma_{2}+\omega_{0}^{-1} \omega_{2} \frac{\partial}{\partial t}\right) \tilde{\psi}_{1}\right. \\
\left.+R_{0}^{-1} P \theta_{1 I}\left(\sigma_{2}+\omega_{0}^{-1} \omega_{2} \frac{\partial}{\partial t}\right) \tilde{\theta}_{1}\right\rangle+R_{0}^{-1} R_{2}\left\langle\theta_{1 I} \Delta_{2} \tilde{v}_{1}\right\rangle \\
+\left\langle\left(V_{1 I} \boldsymbol{\delta}+\psi_{1 I} \epsilon\right) \cdot\left(\tilde{\mathbf{u}}_{1} \cdot \nabla \mathbf{u}_{2}+\mathbf{u}_{1} \cdot \nabla \tilde{\mathbf{u}}_{2}+\tilde{\mathbf{u}}_{2} \cdot \nabla \mathbf{u}_{1}+\mathbf{u}_{2} \cdot \nabla \tilde{\mathbf{u}}_{1}\right)\right. \\
\left.+R_{0}^{-1} P \theta_{1 I}\left(\tilde{\mathbf{u}}_{1} \cdot \nabla \theta_{2}+\mathbf{u}_{1} \cdot \nabla \tilde{\theta}_{2}+\tilde{\mathbf{u}}_{2} \cdot \nabla \theta_{1}+\mathbf{u}_{2} \cdot \nabla \tilde{\theta}_{1}\right)\right\rangle=0, \quad I=1,2 .
\end{gathered}
$$

The triple products in (17) contain the horizontal averages of the functions $\cos \left[\left(\mathbf{k}_{n}+\mathbf{k}_{m}+\mathbf{k}_{l}-\mathbf{k}_{p}\right) \cdot \mathbf{r}\right]$ and $\cos \left[\left(\mathbf{k}_{n}-\mathbf{k}_{m}+\mathbf{k}_{l}+\mathbf{k}_{p}\right) \cdot \mathbf{r}\right]$ which are non zero only in the following cases respectively:

$$
\text { (l) } p=n, \quad l=-m ; \quad \text { (II) } l=-n, \quad p=m, \quad p \neq n
$$

and

$$
\text { (I) } p=n, \quad l=m ; \quad \text { (II) } l=-n, \quad p=m, \quad p \neq-n \text {. }
$$

Using (18) in (17a) and after some considerable algebra, we find the following results for the leading order terms:

$$
\begin{gathered}
R_{2}=4^{-1} p^{2} a^{6}, \\
\omega_{2}=\left(16 \omega_{0}\right)^{-1} \pi^{2} a^{4}-\left(6 a^{4}\right)^{-1} P \omega_{0} R_{2}, \\
c_{-1}=c_{1}= \pm 1 / 2 .
\end{gathered}
$$

Using (16) and (19a) in the expansion for $R$ given in (6), we find that the oscillatory motion occurs at $R>R_{0 c}$. Hence, there is no subcritical instability and the motion is supercritical. Using (18) and (19c) in (17b) and after some 
lengthy algebra, we find the following system of equations for the leading order terms

$$
\begin{gathered}
S_{0} \tilde{c}_{1}=3 \tilde{E}_{-1}, \quad S_{0} \tilde{c}_{-1}=3 \tilde{E}_{1}, \\
6 \tilde{c}_{-1}+S_{0} \tilde{E}_{1}=\tilde{c}_{1}, \quad 6 \tilde{c}_{1}+S_{0} \tilde{E}_{-1}=\tilde{C}_{-1},
\end{gathered}
$$

where

$$
S_{0}=8 \omega_{0}\left(2 a^{2} \sigma_{2}-3^{-1} R_{2}\right)\left(\pi a^{3}\right)^{-2} .
$$

Equations $(20 \mathrm{a}, \mathrm{b})$ and $(20 \mathrm{c}, \mathrm{d})$ are resulted from $(17 \mathrm{~b})$ for $i=1$ and $i=2$, respectively. For the special disturbances for which $\tilde{E}_{-1}=\tilde{E}_{1}=0$, then $(20 \mathrm{a}, \mathrm{b})$ imply that

$$
S_{0}=0
$$

which holds for non-trivial solutions $\tilde{c}_{-1}$ and $\tilde{c}_{1}$. Similarly if $\tilde{c}_{-1}=\tilde{c}_{1}=0$, then (20c, d) imply (21) which now holds for nontrivial solutions $\tilde{E}_{-1}$ and $\tilde{E}_{1}$. In the general case, $(20)$ yields

$$
S_{0}^{4}+36 S_{0}^{2}+315=0
$$

which is the characteristic equation for (20). The solutions to (22) are

$$
S_{0}= \pm(21)^{1 / 2} I, \quad S_{0}= \pm(15)^{1 / 2} I .
$$

It is clear that if (21) holds, then (20e) implies that

$$
\sigma_{2}=R_{2}\left(6 a^{2}\right)^{1 / 2}
$$

However if (23) holds, then (20e) implies that

$$
\begin{aligned}
& \sigma_{2}= \pm\left(16 \omega_{0}\right)^{-1}(21)^{1 / 2} \pi^{2} a^{4} I+R_{2}\left(6 a^{2}\right)^{-1}, \\
& \sigma_{2}= \pm\left(16 \omega_{0}\right)^{-1}(15)^{1 / 2} \pi^{2} a^{4} I+R_{2}\left(6 a^{2}\right)^{-1} .
\end{aligned}
$$

Since either the expression (24) or the real parts of the expressions (25) for $\sigma_{2}$ are positive, we have shown that the overstable rolls are unstable.

So far, the analysis has been restricted to disturbances whose wavenumber vectors coincided with the basic vector of the overstable two-dimensional motion. We now would like to consider the analysis for the disturbances whose wavenumber vectors do not coincide with the basic vector of the original oscillatory rolls. Defining the disturbance vector $\mathbf{k}_{r}$, the leading order terms of the equations for $\sigma_{2}, \tilde{E}_{n}$ and $\tilde{c}_{n}$, after some lengthy algebra, can be written in the following forms:

$$
\begin{gathered}
\left(\frac{14}{3} A_{n 1}+B_{n 1}\right) \tilde{c}_{-n}+\left(\frac{14}{3} A_{n 1}+S_{1}\right) \tilde{c}_{n}+m \tilde{E}_{n}=0, \quad n=-r, r, \quad \\
\left(2 \omega_{0} a^{-2} A_{n 1}-m\right) \tilde{c}_{-n}+2 \omega_{0} a^{-2} A_{n 1} \tilde{c}_{n}+S_{1} \tilde{E}_{-n}+B_{n 1} \tilde{E}_{n}=0, \quad n=-r, r,
\end{gathered}
$$


where

$$
\begin{aligned}
m & =6^{-1} P \omega_{0} R_{2}+a^{4} \omega_{2}, \quad S_{1}=a^{4} \sigma_{2}-6^{-1} a^{2} R_{2}, \\
B_{n 1} & =(8 \tau)^{-1} a^{10} \phi_{n 1} H_{n 1} \\
A_{n 1} & =(32 \tau)^{-1} a^{10}\left(1-\phi_{n 1}^{2}\right)^{-1} \phi_{n 1} H_{n 1} \\
\phi_{n 1} & =a^{-2}\left(\mathbf{k}_{n} \cdot \mathbf{k}_{1}\right), \quad H_{n 1}=a^{-2}\left(\mathbf{k}_{n} \times \mathbf{k}_{1}\right) .
\end{aligned}
$$

For the special disturbances for which $\tilde{E}_{-r}=\tilde{E}_{r}=0$, then (26a) and (27) yield the following solutions for $\sigma_{2}$ :

$$
\begin{gathered}
\sigma_{2}=a^{-4} B_{r 1}+6^{-1} a^{-2} R_{2}, \\
\sigma_{2}=-a^{-4}\left(28 A_{r 1} / 3+B_{r 1}\right)+6^{-1} a^{-2} R_{2} .
\end{gathered}
$$

Using (27) in (28) and the fact that $A_{n 1}$ (or $B_{n 1}$ ) can have either sign, we find that $\sigma_{2}$ becomes positive for all possible values of $\phi_{r 1}\left(-1 \leqslant \phi_{r 1} \leqslant 1\right)$. The growth rate $\sigma_{2}$ has its largest positive values for $\phi_{r 1}^{2}=1$. Similarly if $\tilde{c}_{r}=\tilde{c}_{r}=0$, then (26b) and (27) yield the following solutions for $\sigma_{2}$ :

$$
\begin{aligned}
& \sigma_{2}=a^{-4} B_{r 1}+6^{-1} a^{-2} R_{2}, \\
& \sigma_{2}=-a^{-4} B_{r 1}+6^{-1} a^{-2} R_{2} .
\end{aligned}
$$

Again $\sigma_{2}$ given by (29) is real and positive for all possible values of $\phi_{r 1}$, but the maximum growth rates are now reached for $\phi_{r 1}^{2}=12$. For the general disturbances of the type (12), (26) yields

$$
\begin{aligned}
\left(S_{1}^{2}-2 B_{r 1} S_{1}\right. & \left.+B_{r 1}^{2}+m^{2}\right) \\
\times\left[S_{1}^{2}+\left(28 A_{r 1} / 3+2 B_{r 1}\right) S_{1}\right. & +B_{r 1}\left(28 A_{r 1} / 3+B_{r 1}\right) \\
& \left.+m\left(m-4 a^{-2} \omega_{0} A_{r 1}\right)\right]=0 .
\end{aligned}
$$

Using (27) and (30), we find the following solutions for $\sigma_{2}$ :

$$
\begin{gathered}
\sigma_{2}=\left(6 a^{2}\right)^{-1} R_{2}+a^{-4} B_{r 1} \pm a^{-4} m I \\
\sigma_{2}=\left\{-\left(14 A_{r 1} / 3+B_{r 1}\right)\right. \\
\left. \pm\left[196 A_{r 1}^{2} / 9-m^{2}+4 a^{-2} \omega_{0} m A_{r l}\right]^{1 / 2}+a^{2} R_{2} / 6\right\} a^{-4} .
\end{gathered}
$$

Equation (31) indicates that $\sigma_{2}$ can have complex values, but the real part of $\sigma_{2}$ is positive and reaches its maximum value for $\phi_{r 1}^{2}=1$. A simple calculation shows that the maximum value of the growth rate of these latter disturbances is largest among the growth rates of all the disturbances discussed in this section. 


\section{Discussion}

The expression (19a) for the coefficient $R_{2}$ which is the largest nonlinear correction term to $R_{0 c}$ in the expansion (6) for $R$ (since $R_{1}=0$ by (16)) is clearly positive. Hence, the oscillatory motion of the two-dimensional rolls is supercritical and occurs at $R>R_{0 c}$. There is, therefore, no subcritical instability. This important result that the finite amplitude instability does not occur in the present problem indicates that the fluid is not able to release sufficient amounts of potential energy to compensate for the significant viscous dissipation resulting from the finite amplitude growth. No disturbance can, therefore, grow in the present problem to offset the effects of rotation and to maintain the convective flow when the Rayleigh number is reduced below its critical value according to linear theory.

The expression for the heat transported by the convective rolls, $\mathrm{H}^{\mathrm{c}}$, can be evaluated using (9) and (19a). We have

$$
\begin{aligned}
H^{c} & =p^{2} R^{-1}\langle\theta \mathbf{u} \cdot \lambda\rangle \cong \mu^{2} a_{c}^{2} P^{2} R^{-1}\left\langle v_{1} \theta_{1}\right\rangle \\
& \cong a_{c}^{2} P^{2}\left(R R_{2}\right)^{-1}\left(R-R_{0 c}\right)\left\langle v_{1} \theta_{1}\right\rangle=4\left(R-R_{0 c}\right) / R a_{\imath}^{2} .
\end{aligned}
$$

Using the expression for $a_{c}$ given by (11) in (32), we find that the heat transported by convection is quite small and decreases with increasing $P$ or $\tau$. These results are expected since it is known [2] that oscillatory convection is not quite effective at transporting heat and that viscous or coriolis forces have stabilizing effects on the convective flow.

The expression (19b) for the coefficient $\omega_{2}$ which is the largest nonlinear correction to $\omega_{0 c}$ in the expansion (6) for $\omega$ (since $\omega_{1}=0$ by (16)) implies that the frequency $\omega$ is a function of the amplitude $\mu$. Using (19a) in (19b), we find that to the leading order term

$$
16 \omega_{0} \omega_{2}=\pi^{2} a_{c}^{4} .
$$

This result means that the finite amplitude effects on the frequency has the same sign as the linear frequency $\omega_{0 c}$. Hence for supercritical motion $R>R_{0 c},|\omega|>$ $\left|\omega_{0 c}\right|$. Nonlinear processes, therefore, cause the period of overstable oscillations to become smaller than that in the linear regime. Using (6), we find that to the second order in $\mu$

$$
\mu^{2} \cong R_{2}^{-1}\left(R-R_{0 c}\right) \cong \omega_{2}^{-1}\left(\omega-\omega_{0 c}\right) .
$$

Equation (34) shows the effects of the nonlinear change in the frequency on the amplitude of convection and subsequently on the heat transport. The change in the frequency causes $R_{2}$ to become positive and the motion becomes stable to finite amplitude perturbations. 
Various kinds of disturbances which lead to instability of the oscillatory rolls were discussed in Section 2. Some of these disturbances which may or may not be along the oscillatory rolls have the same period of oscillations as that of the overstable motion. The nonlinear effect on the frequency of some of the disturbances which are along the oscillatory rolls is different from the nonlinear effect on the frequency of the overstable motions. However only some of the disturbances which are along the original rolls and have the same period of oscillations as that of the overstable motion are the most dangerous ones. That is, they have the largest positive growth rate among all the disturbances discussed in Section 2, and are therefore amplified and will be predominant in the subsequent nonlinear development of the time dependent flow field.

The growth rates of all the disturbances discussed in Section 2 are almost independent of the period of the rolls' oscillations. Although oscillatory motion is preferred in the present study, it is known to be inefficient at transporting heat. Since the heat transported by convection is proportional to $\mu^{2}$, the frequency of the rolls' oscillations is indeed expected to have no significant effect on the growth of the amplitude of the disturbances.

The instability of the overstable motion and the amplification of the disturbances having the maximum growth rate discussed in Section 2 are due to the toroidal component $\epsilon \psi$ of $\mathbf{u}$ given in (la) which dominates over the poloidal components $\delta v$ of $\mathbf{u}$ given in (1a) for asymptotically large $\tau$. This interesting mathematical result seems to have a general validity (beyond the conditions (8)) for sufficiently large parameters $\tau$ and $P^{-1}$ and can be followed from the following discussion. It can be shown from (27) and (31) that the real part of $\sigma_{2}$ can be written as $D_{r 1}^{s}+D_{r 1}^{a}$, where $D_{r 1}^{s}$ and $D_{r 1}^{a}$ are in general functions of $\phi_{r 1}, H_{r 1}$, $\tau$ and $P$. Now, $D_{r 1}^{s}$ is symmetric with respect to subscripts $r$ and $l$ and satisfies the properties $D_{r 1}^{s}=D_{1 r}^{s}=D_{-r, 1}^{s}$. Further $D_{r 1}^{a}$ is anti-symmetric with respect to subscript $r$ and 1 and satisfies the following properties $D_{r 1}^{a}=-D_{1 r}^{a}=-D_{-r, 1}^{a}$. It can also be shown (details are not given here) that the symmetric function $D_{r 1}^{s}$ is due to the poloidal component $\delta v$, while the anti-symmetric function $D_{r 1}^{a}$ is due to the toroidal component $\epsilon \psi$. When $\tau$ and $P^{-1}$ are sufficiently large, $D_{r 1}^{a}$ is much larger in magnitude than $D_{r 1}^{s}$. The sign of the growth rate of the disturbances is then the same as the sign of $D_{r 1}^{a}$. Due to the anti-symmetries of the function $D_{r 1}^{a}$ discussed above, a disturbance whose wave number vector is either $\mathbf{K}_{r}$ or $\mathbf{K}_{-r}$ causes the function $D_{r 1}^{a}$ to become positive and consequently the overstable motion becomes unstable. Using (1), we find that that vertical vorticity $(\nabla \times u) \cdot \lambda$ equals $-\Delta_{2} \psi$ and thus depends only on the toroidal component $\epsilon \psi$ or $u$. The instability of the overstable motion discussed above is caused by the nonlinear terms in the momentum equations and is due to $\epsilon \psi$ that generates a significant nonlinear vorticity which counteracts the vorticity generated by the external constraint of rotation. 
The assumption (8) made earlier in Section 2 for a rapidly rotating layer implies of course that $P$ is small. Mercury is a typical small Prandtl number fluid which satisfies the condition (8). For mercury, $P=0.025$, we find from (8) the range for $\tau$ for which the results of the present study apply

$$
40 \ll \tau \ll(1.0112) \times 10^{4} .
$$

Veronis [9] considered the problem of overstable convective motion in the form of standing waves in his study of small amplitude convection in a rotating low Prandtl number fluid. He used a modified version of the method of Malkus and Veronis [6] to determine the weakly nonlinear behavior of the flow and evaluated numerically the finite amplitude effects for the convection cells. For mercury, he found, for example, that in the range (34), $H^{c}$ increases with $\tau$ for oscillatory rolls. This result does not agree with experimental evidence, [7], as well as with our finding. He also found that $H^{c}$ is quite small for the oscillatory rolls and that the flow of mercury is not unstable subcritically. These results agree with our findings.

Rossby [7] considered the problem of rotating mercury in his experimental study of Benard convection. He observed a horizontal buckling of rolls that eventually broke up into vorticities at sufficiently large $\tau$. Mercury was found to be unstable and unsteady. He also found that $H^{c}$ was quite small and decreases with increasing $\tau$. These results are all in qualitative agreement with our present results. An evidence found in [7] which is not predicted by the present study is the existence of finite amplitude oscillatory instability for $134<\tau<316$. It is, however, quite possible that the value of $\tau$ in this range is not large enough to satisfy (34).

The above discussions indicate the need for further studies in the subject. Although the present study is the first investigation of the nonlinear overstable convection with its instability, its range of validity is rather restricted due to condition (8) and the assumption that $R$ is close to $R_{0 c}$. There are still many problems which need to be studied particularly for the ranges of $\tau$ and $P$ other than those considered in this paper.

\section{References}

[1] F. H. Busse and R. M. Clever, "Nonstationary convection in a rotating system", in Recent developments in theoretical and experimental fluid mechanics (eds. U. Muller, K G. Roerner and B. Schmidt), (Springer-Verlag, 1979), 376-385.

[2] S. Chandrasekhar, Hydrodynamic and hydromagnetic stability (Oxford University Press, 1961).

[3] R. M. Clever and F. H. Busse, "Nonlinear properties of convection rolls in a horizontal layer rotating about a vertical axis", J. Fluid Mech. 94 (1979), 609-627. 
[4] G. Kuppers, "The stability of steady finite amplitude convection in a rotating fluid layer", Phys. Lett. A 32 (1970), 7-8.

[5] G. Kuppers and D. Lortz, "Transition from laminar convection to thermal turbulence in a rotating fluid layer", J. Fluld Mech. 35 (1969), 609-620.

[6] W. V. R. Malkus and G. Veronis, "Finite amplitude cellular convection", J. Fluid Mech. 4 (1958), 225-260.

[7] H. T. Rossby, "A study of Benard convection with and without rotation", J. Fluid Mech. 36 (1969), 309-335.

[8] A. Schluter, D. Lortz and F. H. Busse, "On the stability of steady finite amplitude convection". J. Flurd Mech. 23 (1965), 129-144.

[9] G. Veronis, "Cellular convection with finite amplitude in a rotating fluid", J. Flud Mech. 5 (1959), 401-435. 